\title{
40. MICROPALEONTOLOGICAL INVESTIGATIONS OF SEDIMENTS FROM SITES 379, 380, AND 381 OF LEG 42B
}

\author{
Musat Gheorghian, Institute of Geology and Geophysics, Bucharest, Romania
}

\section{INTRODUCTION}

Three hundred and thirty-nine samples from Black Sea Sites 379, 380, and 381 of Leg 42B of the Deep Sea Drilling Project have been sent to the author by Drs. Kenneth Hsü and Stephen Percival, participants on this cruise. All samples have been investigated micropaleontologically, but only 119 contain microfossils.

\section{ORIGIN OF THE POPULATIONS OF FORAMINIFERS}

As a result of the research on the Neogene foraminifers of the Black Sea, it is established that since middle Miocene time they have evolved independent of the Mediterranean Sea. The Black Sea sediments that accumulated within this time interval generally contain specimens of eurihaline foraminifers associated with endemic species or subspecies adapted to reduced salinity. There are stenohaline foraminifers as well, proving that during the Mio-Pliocene and Quaternary, migrations of stenohaline faunas from the Mediterranean basin took place and that they adapted to the reduced salinity characteristic of the Black Sea. This is, for example, the case for the specimens of Bolivina inflata and B. spiralis from Site 381 (Cores 35, 37) situated near the Bosporus area, and for the specimens of Laryngosigma semitecta and $L$. williamsoni from the Recent sediment of the shelf (Mikalevici, 1968; Gheorghian, 1974). Their adaptation is manifested by a reduction of size and of wall thickness up to a transparent pellicle stage.

Another group of benthic foraminifers (Helenina anderseni, Elphidium pulvereum, E. haagensis) found in both DSDP cores and Recent sediments of the western shelf of the Black Sea are known from estuarine sediments or from the beach sands of the North Sea and English Channel. For the moment, their presence in the Black Sea material cannot be explained.

Except for the above-mentioned groups, the foraminiferal microfauna of the three sites consists predominantly of numerous specimens of Ammonia beccarii, A. parkinsoniana, A. perlucida, A, tepida, A. viennensis-compacta, and Elphidium advenum ponticum, E. macellum, E. reginum, Glabratella $\mathrm{cf}$. G. kartvelica.

The absence of planktonic foraminifers in the Neogene to Recent sediments is notable; however, occasional isolated specimens of Globerina and Globigerinoides have been recorded.

Mysid statoliths of Paramysis mihaii were also found. In spite of their until recent disputable systematic position, they have been used as marker fossils for the upper Volhynian-lower Bessarabian of the Central and Eastern Paratethys. The micropaleontological investigations based on foraminifers and mysid statoliths prove that the three drillings ${ }^{1}$ penetrated Quaternary, Pliocene, and upper Miocene sediments.

Foraminifers are missing in the Postglacial sediment but are abundant and diverse in Würm, Riss, Mindel, and upper Miocene (see Tables 1-3).

\section{REFERENCES}

Gheorghian, M., 1974. Distribution pattern of benthonic foraminifera on Continental Shelf of Black Sea off Rumanian Shore: Am. Assoc. Petrol. Mem. 20, p. 411-418.

Mikhalevici, V.I., 1968. Foraminifera.In Fodeanitkii, V.A. (Ed.), Opredjivuscic bezpozvonocinie, v. 1, p. 9-21.

\section{LIST-FORAMINIFERS}

\section{APPENDIX-FAUNAL REFERENCE}

Ammonia beccarii (Linne): Belford, D.J., 1966, Australia Bur. Min. Res. Bull. 79, p. 108-110, pl. 19, fig. 2-8.

Ammonia parkinsoniana (d'Orbigny)-Streblus parkinsonianus (d'Orbigny): Hofker, J., 1971, Stud. Foram. Curac. Caraib. Isl., v. 127 , p. $51-52$, fig. $138-153$, Holland.

Ammonia perlucida (H. Allen and Earland)-"Rotalia" perlucida $\mathrm{H}$. Allen and Earland: Albani A.D., 1968, Cushman Found. Foram. Res. Contrib., v. 19, p. 110, fig. 12, 16.

Ammonia sikokuensis (Ishizaki): Huang, T., 1964, Micropaleontology, v. 10 , p. 54 , pl. 1, fig. 1.

Ammonia tepida (Cushman)-Streblus beccarii (L.) var. tepida Cushman: Todd, R., 1957, U.S. Geol. Surv. Prof. Pap. 280-H, p. 278 , pl. 91 , fig. 5a-c.

Ammonia viennensis-compacta (Hofker)-Rosalina viennensis d'Orbigny:d'Orbigy, A., 1846, Foram. Foss. Wien, p. 177-178, pl. X, fig. 22-24. Streblus compactus Hofker, J., 1971, Stud. Foram. Curaç. Caraib. Isl. v. 127, p. 43-50, fig. 114-137, Holland.

Bolivina inflata H. Allen and Earland: H. Allen and Earland, 1913, Roy. Irish. Acad., Proc. XXXI, p. 68, pl. IV, fig. 16-19.

Bolivina siralis Cushman: Cushman, J.A., 1926, Cushman Lab. Foram. Res. Contrib. v. II, p. 31, pl. IV, fig. 6.

Cribroelphidium poeyanum (d'Orbigny)-Elphidium poeyanum (d'Orbigny):Cushman, J.A., 1939, U.S. Geol. Surv. Bull., v. 191, p. 54 , pl. 14, fig. $25,26$.

Elphidium advenum ponticum Dolgopolskaya and Pauli, 1931, Trav. Stat. Biol. Karadagh, v. 4, p. 36, pl. III, fig. 14.

Elphidium alvarezianum (d'Orbigny)-Polystomella alvareziana d’Orbigny:1839, Voyage Amér. Mérid., v. V, p. 31, pl. III, fig. $11,12$.

Elphidium crispum (Linné)-Polystomella crispa (L.): d'Orbigny, A., 1846, Foram. Foss. Wien, p. 125, pl. VI, fig. 9-14.

Elphidium incertum (Williamson): Cushman, J.A., 1939, U.S. Geol. Surv. Prof. Pap. 191, p. 57, pl. XV, fig. 21-24.

'Editorial note: this comment apparently should not apply to Site 379. 
Elphidium macellum (Fichtel and Moll)—Nautilus macellus Ficht. Moll: 1978 , Test. Micr., p. 66 , var. $a$, pl. X, fig. e-g; var. $\beta$, pl. X, fig. h-k.

Elphidium pulvereum Todd: 1948,Rept. Swedish Deep-Sea Exped., v. 8 , p. 210 , pl. 1 , fig. $19-20$.

Elphidium reginum (d'Orbigny)-Polystomella regina d'Orbigny, :1846, Foram. Foss. Wien, p. 129, pl. VI, fig. 23, 24.

Glabratella kartvelica Krash.:Krasheninikov in P.P. Zhizhcenko, 1959, Atlas Sprednemiot. faunî Sev. Kawkaz. Krîm, p. 49-50, pl. VIII, fig. la, b, v.

Helinina anderseni (Warren)-Pseudoeponides anderseni Warren: 1957, Contrib. Cushman Found. Foram. Res., v. VIII, p. 29-40, pl. 4, fig. 12-15.

Lagena lateralis pontica Mikhalevich: 1968, Opred. faunî Ciornogo morea i Azovskogo morei, pl. 18, pl. V, fig. 2.

Massilina secans (d'Orbigny)-Quinqueloculina secans d'Orbigny, A., 1826, An. Sci. Nat., v. VII, p. 303.

Nonion depressulum (Walker and Jacob): Cushman, J.A., 1939, U.S. Geol. Surv. Prof. Pap. 191, p. 20, pl. 5, fig. 22-25.

Protelphidium martkobi (Bogdanovich): 1947, Microfauna Kawkaza, Embî i Srednei Azii, p. 30, pl. IV, fig. 4a-c.
Protelphisium subgranosus (Egger)-Nonion subgranosus (Egger): Macarovici, N. and Ionesi, B., 1966, An. St. Univ. Cuza Iaşi, v. 12, p. 91-92, pl. II, fig. 2 .

Pyrgo williamsoni Silvestri and Zangheri-Biloculina ringens Lamk. in Williamson Recent British Foram., 1858, p. 78, pl. VI, fig. 169-170, pl. VII, fig. 171-176.

Quinqueloculina ersaconica Krash.: Krasheninikov in P.P. Zhizhcenko, 1959, Atlas Srednemioț. faunî Sev. Kawkaz. Krîm, p. 76-77, pl. VII, fig. 1a, b.

Quinqueloculina haidingeri d'Orbigny, 1846, Foram. Foss. Wien, p. 289 , pl. XVIII, fig. 13-15.

Quinqueloculina laevigata d'Orbigny: 1826, Ann. Sci. Nat. VII, p. 301.

Quinqueloculina seminulum (Linné)—Serpula seminulum Linné, 1758, ed. X, p. 786.

\section{MYSIDAE}

Paramysis mihaii Voicu: 1974, Geol. Zborn. Geol, Carpatica, XXV no. 2 , p. 231 , fig. $2,4,6$.

TABLE 1

Foraminifers From Site 379

\begin{tabular}{|c|c|c|c|c|c|c|c|c|c|c|c|c|c|c|c|c|c|}
\hline \multirow[b]{2}{*}{ 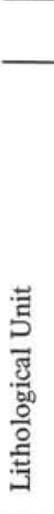 } & \multirow[b]{2}{*}{$\begin{array}{l}\text { Sample } \\
\text { (Interval } \\
\text { in } \mathrm{cm} \text { ) }\end{array}$} & \multicolumn{10}{|c|}{ Foraminifers } & \multicolumn{4}{|c|}{ Reworked } & \multicolumn{2}{|c|}{ Varia } \\
\hline & & 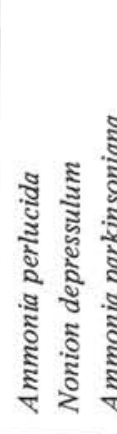 & 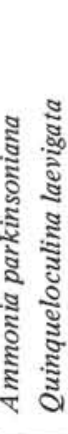 & 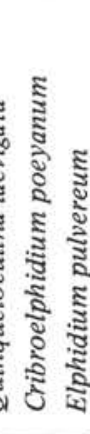 & 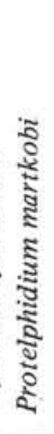 & 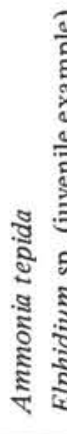 & 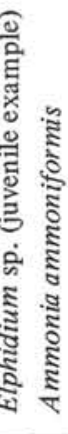 & 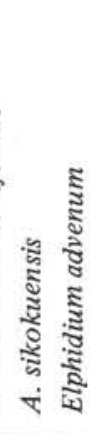 & 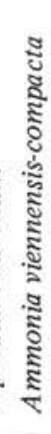 & 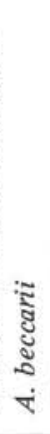 & 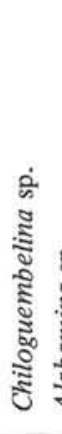 & 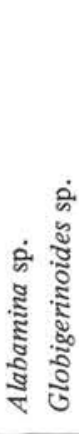 & 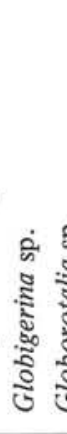 & 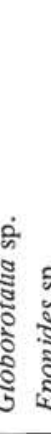 & 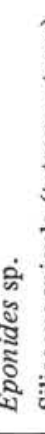 & 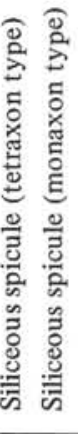 & 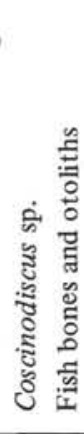 \\
\hline 3 & $1-4,40-42$ & & & & & & & & & & & & & & & & + \\
\hline 4 & $8-4,37-39$ & & & & & & & & & + & & & & & & + & \\
\hline 4 & $8-5,64-66$ & & & & & & & & & + & + & & & & & + & + \\
\hline 4 & $9-1,120-122$ & + & + & & + & & & & & + & & & & & & + & + \\
\hline 4 & $9-5,111-113$ & & & & & & & & & + & & & & & & & \\
\hline 4 & $10-2,100-102$ & & & & & & & & & + & & & & & & & \\
\hline 4 & $10-4,75-77$ & ++ & & & & & & & & + & & & & & & & \\
\hline 4 & $10-6,114-116$ & & & & & & & & & + & & & & & & & \\
\hline 4 & $10, \mathrm{CC}$ & ++ & ++ & & & & & & & + & & & & & & + & + \\
\hline 4 & $11-5,15-17$ & & & & + & & & & & + & & + & & & & & \\
\hline 4 & $11-5,26-27$ & & + & ++ & & + & & & & + & & + & & & & & \\
\hline 6 & $13-5,91-93$ & & & & & + & + & & & + & & & & & & & \\
\hline 6 & $14-6,22-24$ & & & & & & & & & & & & & & + & & \\
\hline 6 & $15-2,48-50$ & & & & & & + & & & & & & & & & & \\
\hline 6 & $16-1,120-122$ & & & & & & & ++ & & + & & & & & & & \\
\hline 6 & $16-3,47-49$ & & & & + & & & & & & & + & & + & + & & \\
\hline 6 & $17-1,89-91$ & & & & & & & & + & & & & & & & & \\
\hline 6 & $21-4,20-22$ & & & & & & & & & & & & + & & & & \\
\hline 9 & $51-2,24-26$ & & & & & & & & & & & & + & + & + & & \\
\hline 9 & $51, \mathrm{CC}$ & & & & & & & & & & & & & & + & & \\
\hline
\end{tabular}


TABLE 2

Foraminifers From Site 380

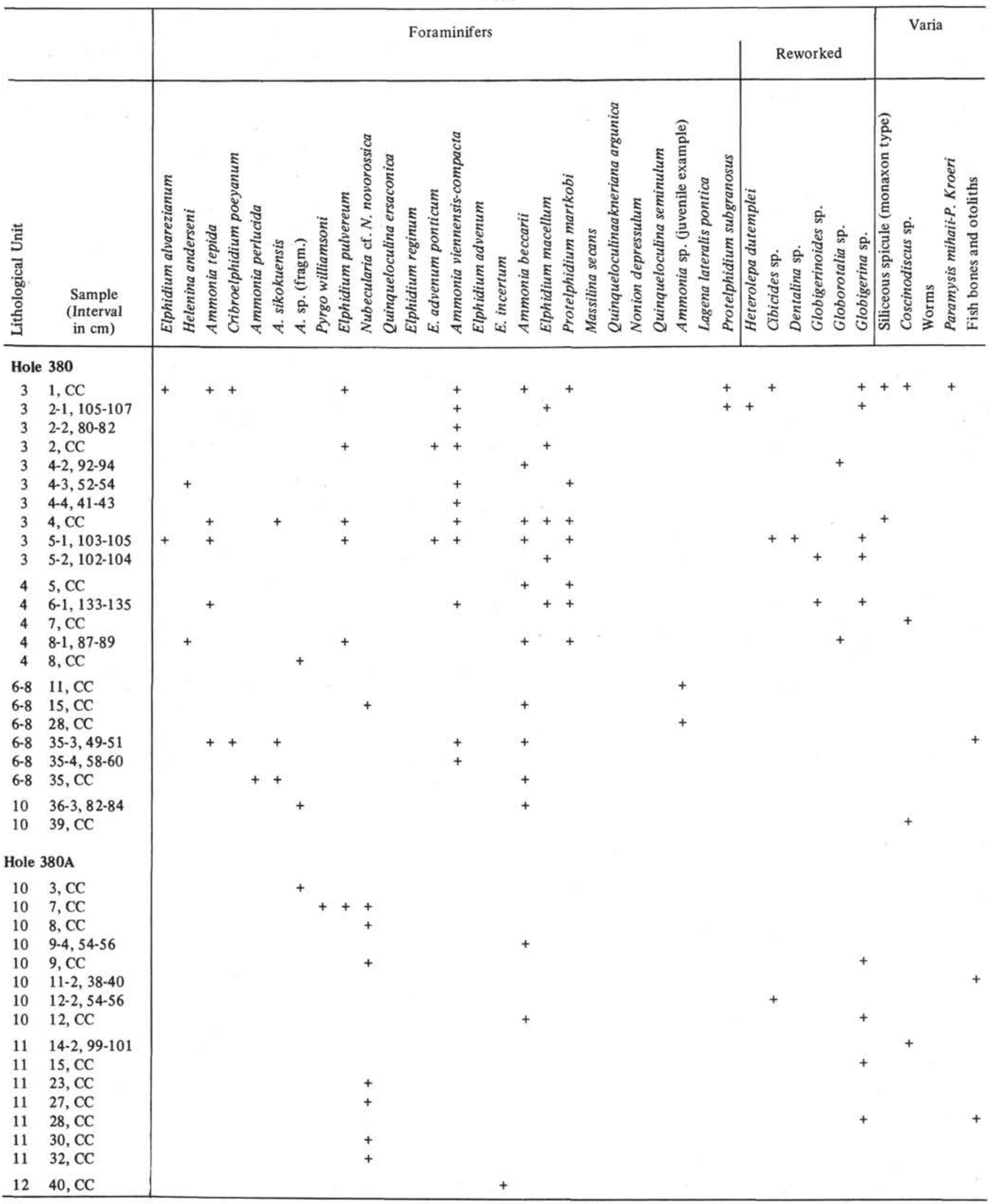


TABLE 2 - Continued

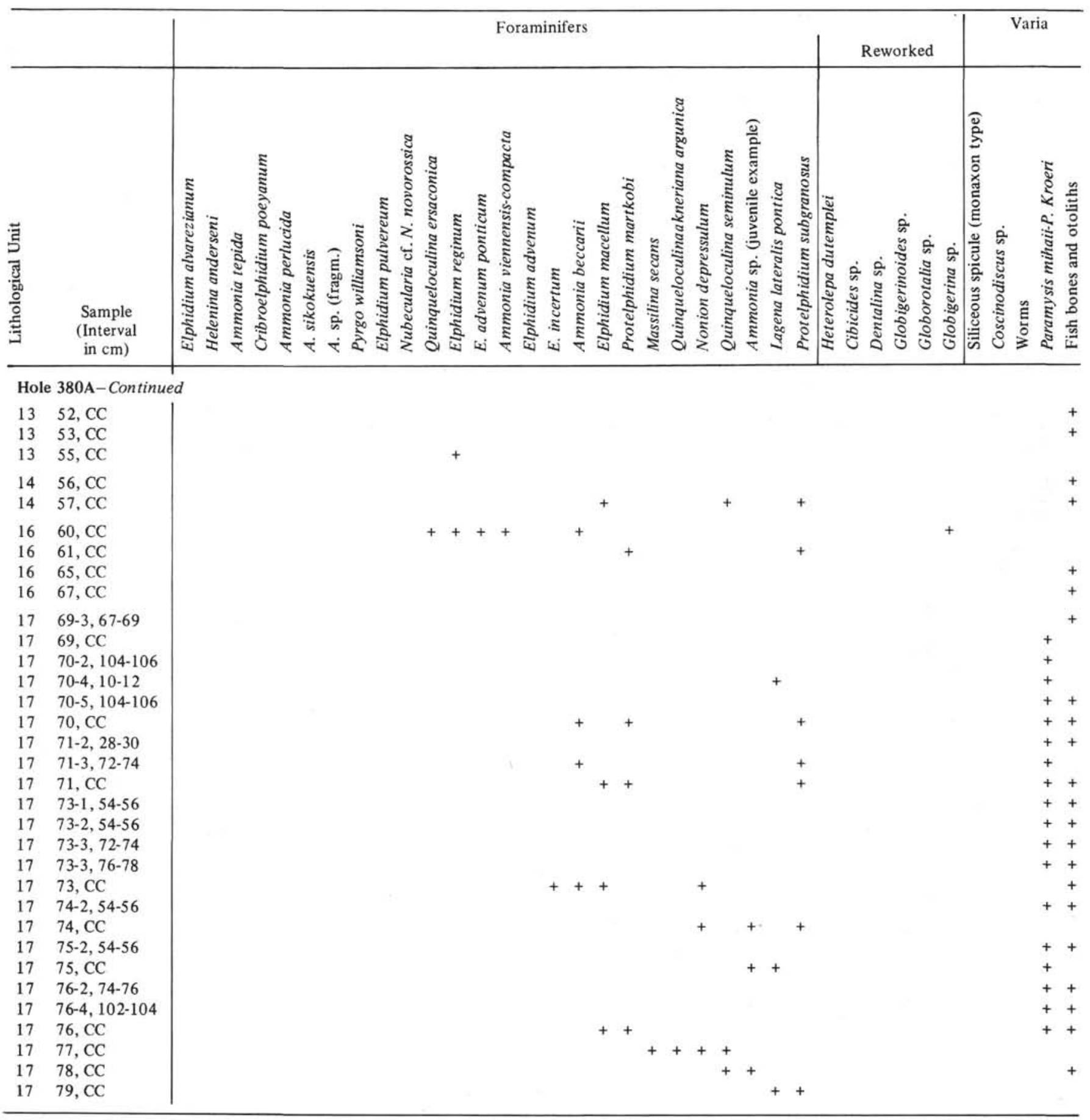


TABLE 3

Foraminifers From Site 381

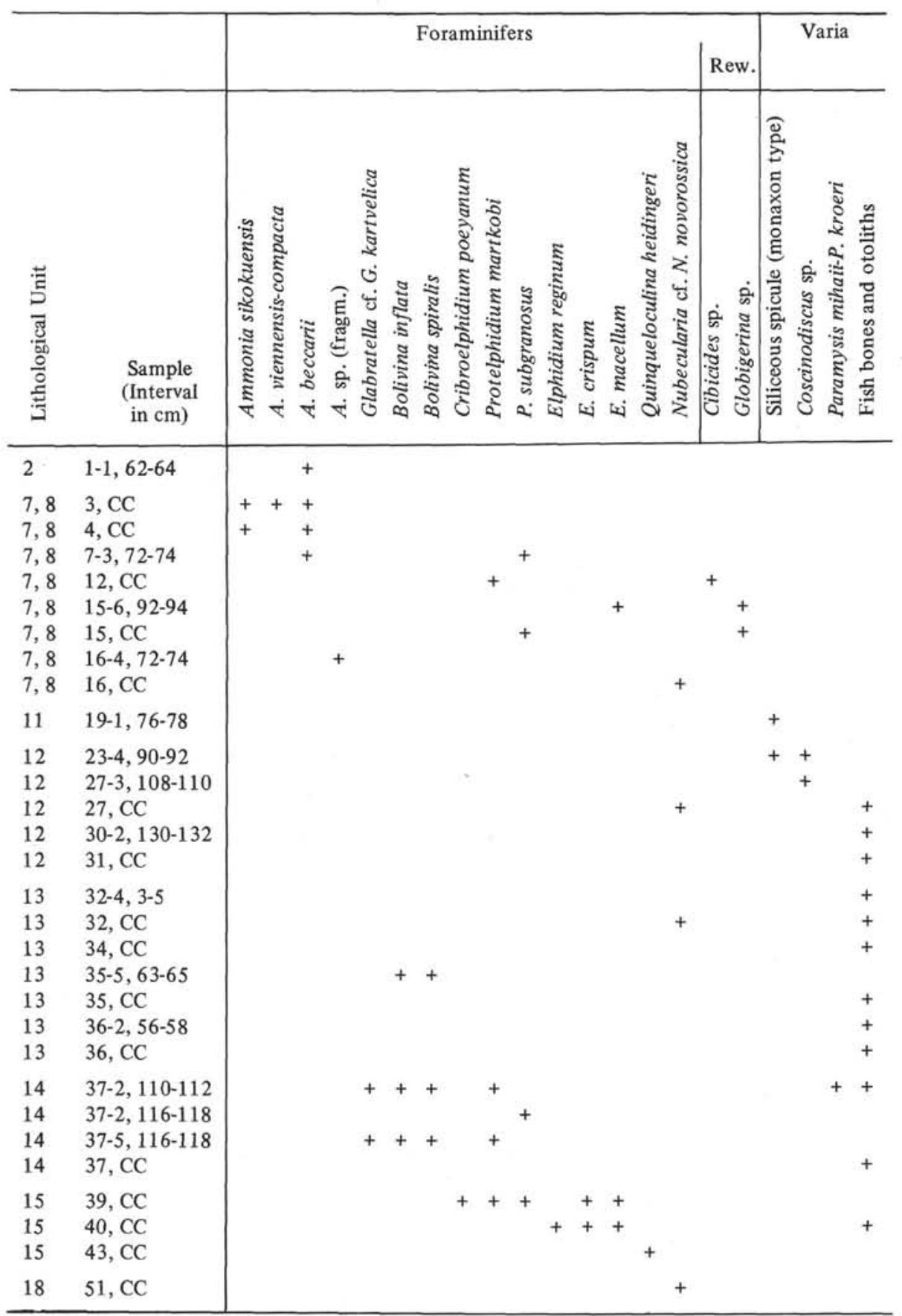

